Meta

Journal des traducteurs

Translators' Journal

\title{
Prolixité et qualité des traductions
}

\section{Denis Juhel}

Volume 44, numéro 2, juin 1999

URI : https://id.erudit.org/iderudit/003275ar

DOI : https://doi.org/10.7202/003275ar

Aller au sommaire du numéro

\section{Éditeur(s)}

Les Presses de l'Université de Montréal

\section{ISSN}

0026-0452 (imprimé)

1492-1421 (numérique)

Découvrir la revue

Citer cet article

Juhel, D. (1999). Prolixité et qualité des traductions. Meta, 44(2), 238-249.

https://doi.org/10.7202/003275ar

\section{Résumé de l'article}

Une traduction est généralement plus longue que l'original. Fait souvent observé, cette longueur ne se laisse pas mesurer aisément, les résultats pouvant ètre contradictoires selon le critère retenu (nombre de mots, de caractères-espaces, ou nombre de phonèmes). De plus, la longueur (de la phrase? du paragraphe ? du texte?) n'est un critère ni nécessaire ni suffisant pour évaluer la qualité de la traduction, bien qu'elle puisse ètre le signe empirique d'un texte perfectible. L'analyse des causes et des effets pervers de la prolixité traductionnelle est suivie de conseils pratiques qui devraient trouver leur application tant dans le cours de révision que dans les premiers enseignements de la traduction. 


\title{
Prolixité et qualité des traductions
}

\author{
denis juhel \\ Université Laval, Québec, Canada
}

\begin{abstract}
RÉSUMÉ
«Une traduction est généralement plus longue que l'original.» Fait souvent observé, cette longueur ne se laisse pas mesurer aisément, les résultats pouvant être contradictoires selon le critère retenu (nombre de mots, de caractères-espaces, ou nombre de phonèmes). De plus, la longueur (de la phrase? du paragraphe? du texte?) n'est un critère ni nécessaire ni suffisant pour évaluer la qualité de la traduction, bien qu'elle puisse être le signe empirique d'un texte perfectible. L'analyse des causes et des effets pervers de la prolixité traductionnelle est suivie de conseils pratiques qui devraient trouver leur application tant dans le cours de révision que dans les premiers enseignements de la traduction.
\end{abstract}

\begin{abstract}
It has long been established that verbose translations are the rule, whatever source or target language is involved. It is not easy to compare the length of a translation with the original text, for different criteria (number of words, number of phonemes, number of lines) give contradictory results. It is neither useful nor relevant to appraise the quality of a translation by its length alone since lengthiness implies much more than mere redundancy. Examples show that correcting verbose translations rarely boils down to cutting words out but rather entails a new formulation for reasons of clarity and fluency. Some tips will help students eradicate translational verbosity at the root when translating or revising a text.
\end{abstract}

L'évaluation des traductions, et plus précisément la recherche de critères qualitatifs, pose une problématique où est mis à contribution le triple champ d'études de la traductologie: la pédagogie, la pratique et la théorie de la traduction. La prolixité (ou foisonnement selon les auteurs), remarquée tant dans les travaux d'étudiants que dans les traductions publiées, peut être un critère intéressant pour le praticien et pour l'enseignant de la traduction dans la mesure où le quantitatif et le qualitatif y sont étroitement imbriqués; c'est tout au moins une hypothèse de départ qu'il convient de vérifier, avec l'espoir de mieux cerner l'objet de la traduction et de mieux délimiter les frontières du sens à traduire.

Quelle que soit la langue de départ ${ }^{1}$, la longueur des traductions par rapport à I'original ( $\leqslant 30 \%)$, constatée depuis fort longtemps, n'a pas fait l'objet jusqu'à ce jour d'études approfondies. En fait, un seul auteur consacre un article entier à la prolixité en traduction: Durieux (1990) ${ }^{2}$.

Bien que l'article de Durieux ne porte que sur la traduction technique, les cinq causes de foisonnement qu'elle distingue peuvent servir de point de départ à mes considérations qui porteront sur la traduction en général, mes exemples étant pris aussi bien dans des textes généraux que spécialisés. 
Avant même d'en examiner les causes, le «constat» du foisonnement mérite d'être revu et relativisé.

Il est donné à tout un chacun, linguiste ou non, de remarquer qu'au Canada (pour ne parler que de ce pays, où environ $80 \%$ des textes français sont des traductions de l'anglais), les traductions françaises sont plus longues que l'original. Parmi les signes les plus évidents, on remarque que la version française occupe un plus grand nombre de pages, ou qu'elle est imprimée en plus petits caractères, que les interlignes sont plus serrés, etc. Une comparaison attentive des deux textes révèle que les contraintes de la mise en page obligent parfois à amputer la version française d'une illustration ou d'un paragraphe et, par conséquent, d'une partie non négligeable de l'information.

\section{PROLIXITÉ ET LONGUEUR}

La manifestation matérielle de la longueur des traductions et sa mesure soulèvent des difficultés d'ordre méthodologique que l'analyse de Durieux n'aborde pas et qui doivent nuancer le constat de longueur. En effet, dire qu'une traduction est plus longue parce qu'elle compte plus de mots que l'original ne peut être qu'une première approximation - à moins de concevoir la traduction comme un exercice de transcodage mot à mot, ce qui n'est certainement pas le cas de Durieux, tenante de la méthode interprétative de la traduction. Sans entrer dans la querelle des linguistes sur la notion même de mot, et pour s'en tenir au comptage des mots que font les logiciels de traitement de texte, il conviendrait de faire la part, partout où c'est possible, entre les contraintes des langues française et anglaise et les choix du discours à la disposition du traducteur (choix stylistiques: paraphrase, redondance ou concision, ellipse, etc.). Sur le plan de la langue, les mots français sont généralement plus longs que les mots anglais (forme canonique du français par rapport à celle de l'anglais: mots de deux syllabes et d'une syllabe respectivement). Ce fait condamne-t-il les traductions françaises à être plus longues que les originaux anglais, même si l'on reconnaît que la traduction n'est pas une opération sur les langues? Convient-il de distinguer entre longueur graphique et longueur phonologique? À partir de quel seuil faudrait-il plus de temps pour lire le texte français parce qu'il a plus de caractères-espaces que le texte anglais? Autant de questions pour faire la part des caractéristiques inhérentes à l'une et l'autre langues, et susceptibles de justifier en partie l'excès de longueur des traductions françaises. Pour ce faire, l'analyse devrait prendre en compte les considérations suivantes, sans tomber dans le piège d'une comparaison mot à mot (l'envers tenace de la traduction mot à mot, condamnée, elle, depuis longtemps) :

1) Nombre de mots dans I'unité de sens: «Au secours! » a bien deux fois plus de mots que son équivalent anglais «H elp ! », mais est-il d'autre «traduction » ou «façon plus courte» de dire la même chose?

2) Nombre de mots et longueur des mots: c'est-à-dire l'espace qu'ils occupent sur la page. «He concealed the truth » / «I a caché la vérité» : 22 caractères-espaces pour les 4 mots de l'anglais, mais 20 caractères-espaces pour les 5 mots du français!

3) Longueur typographique et longueur phonologique: le foisonnement laisse supposer qu'il faut plus de temps pour lire la traduction française que le texte anglais (encore faudrait-il distinguer entre lecture silencieuse et lecture à voix haute). Or, une transcription phonologique fait très vite apparaître la grande différence entre les 
deux types de comparaison. Je n'y arrive pas et I cannot do it se comparent sur le plan de la graphie comme ayant 5 et 4 mots respectivement, 17 caractères-espaces en français contre 14 en anglais. Si graphiquement le français est donc plus long dans cet exemple, sur le plan phonologique on observel'inverse: /Jniarivpa/ /AikœnO tduit/. II y a 9 phonèmes en français et 10 en anglais (dont 1 diphtongue, ce qui permet de conclure à un temps de phonation mesurablement supérieur en anglais).

Une comparaison exhaustive des locutions figées dans les deux langues ferait apparaitre que le français est ici plus long que l'anglais et là plus court, tantôt sur le plan phonologique, tantôt par sa graphie. Peut-être l'inventaire ferait-il ressortir que c'est telle langue qui l'emporte majoritairement sur l'autre sur le plan de la concision phonologique ou graphique. M ais pour qu'une telleétude serveà «l'évaluation quantitative» des traductions, il faudrait qu'elle rende compte des occurrences dans l'une et l'autre langues. Tâche immense, sinon impossible, d'autant moins pertinente que la fréquence statistique est sans conséquence pour un texte donné, et parce qu'un mot ou un syntagme de l'original n'entraîne pas nécessairement l'apparition de son équivalent dans la traduction (par exemple, dans un texte économique sur le pétrole, là où I'anglais précise chaque fois, dans une vingtaine de lignes, «oil demand», «oil production », «oil shortage», le texte français fera très vite l'ellipse du référent pour s'en tenir à «la demande», «la production », «la pénurie»). La description et la comparaison des langues, pour justes et complètes qu'elles puissent être, ne sauraient prédire si la traduction d'un texte sera plus courte ou plus longue que l'original (quel que soit l'étalon servant à mesurer cette longueur matérielle). Cette conclusion s'impose avec d'autant plus d'évidence que la traduction ne saurait être réduite à la simple recherche d'équivalences de mots ou de syntagmes figés.

L'observation communément admise, au moins depuis Barth (1961), selon laquelle une traduction est toujours plus longue que l'original, quelle que soit la langue de départ ou d'arrivée, invite en toute logique à chercher les causes du foisonnement en traduction en dehors des caractéristiques sui generis des langues. Qui plus est, la simple comparaison quantitative entre le texte d'arrivée (TA) et le texte de départ (TD) n'est ni nécessaire ni suffisante pour poser le diagnostic de prolixité ou, à l'inverse, de «bonne traduction », comme pourraient le laisser croire les commentaires de Durieux sur les exemples de foisonnement («Les chiffres parlent d'euxmêmes: 16 mots en anglais, 20 mots en français, soit 25 pour cent de plus») ou de révisions (excellentes) qu'elle propose: «... 15 mots en anglais et pas un de plus en français».

La «traduction idéale» serait-elle celle qui contient autant, voire moins de mots que l'original ? L'exemple qui suit, relevé sur une boîte de lait en poudre, montre les limites du seul critère quantitatif:

This product is sold by weight and not by volume. The powder can settle or pack down during the shipping and handling. If a measuring cup is used to do the mixing, you may not always obtain exactly the same number of quarts of milk from the contents of this package. (51 mots)

Ce produit est vendu au poids et non au volume. À cause de diverses manipulations le lait écrémé en poudre instantané peut se tasser dans l'emballage. C'est pourquoi le nombre de pintes obtenues en reconstituant le lait peut varier légèrement si l'on se sert d'une tasse à mesurer. (48 mots) 
La version française compte moins de mots que l'original anglais (bien qu'elle occupe quelque 19 caractères-espaces de plus sur la page! Plus courte ou plus longue que l'anglais selon le critère retenu !). Une première révision pourrait la raccourcir davantage en faisant l'économie de instantané, dans l'emballage et en reconstituant le lait:

Ce produit est vendu au poids et non au volume. À cause de diverses manipulations le lait écrémé en poudre peut se tasser. C'est pourquoi le nombre de pintes obtenues peut varier légèrement si l'on se sert d'une tasse à mesurer.

Si les 41 mots de cette deuxième version améliorent un tantinet la première et occupent une demi-ligne de moins que le TD, on est encore loin d'une traduction idiomatique. L'extrême concision de la version révisée (33 mots, soit 35\% de moins que l'anglais, et une ligne de moins) n'est pas le seul fait d'un plus grand élagage; nous reviendrons sur la nature des différences entre cette révision et la traduction d'origine:

Ces granulés sont vendus au poids et peuvent se tasser au cours de la manutention. La quantité de lait reconstitué peut varier selon que les granulés sont dosés au poids ou au volume.

Parce que comparer la longueur du TA à celle du TD pose des difficultés d'ordre méthodologique, au demeurant peu pertinentes, nous venons de le voir, pour l'évaluation qualitative des traductions, il conviendra de s'attacher à rechercher dans les textes d'arrivée les véritables «longueurs intrinsèques», en un mot la verbosité, c'està-dire:

1) Pléonasmes/redondances, calques, etc. pouvant être regroupés sous une seule catégorie: surtraduction ${ }^{3}$.

2) Les maladresses stylistiques et les longueurs non reliées à la traduction proprement dite parce qu'elles n'imitent pas servilement, comme les précédentes, la forme du texte anglais; ce sont des maladresses de rédaction que l'on retrouve fréquemment en situation unilingue et que l'on peut qualifier de «déficit de concision ${ }^{4} »$.

Une fois les «mots en trop » éliminés (et autres remaniements), tout «excès de longueur » (nombre de mots ou de caractères-espaces) qui subsisterait pourra être considéré comme irréductible et mis au compte des caractéristiques de la langue d'arrivée.

Dans le présent article, on l'aura compris, les termes prolixité et longueur (au singulier pour ce dernier) sont pris dans leur sens commun et non pas comme synonymes, le second terme dénotant une réalité objectivement mesurable (bien qu'il existe plus d'un étalon possible, comme nous venons de le voir), mais sans le jugement de valeur sur la qualité du texte d'arrivée qu'implique, à l'inverse, prolixité ou son synonyme verbosité.

\section{CAUSES ET EFFETS DE LA PROLIXITÉ $Q$ uelques exemples}

Dire les choses en plus de mots qu'il n'en faut pour être clair s'explique le plus souvent par l'attraction qu'exerce sur le traducteur la formulation du TD - qu'il reproduit — , quoique la maîtrise insuffisante de la langue d'arrivée (LA) puisse être 
également invoquée. Dans ce dernier cas se rajouteront des fautes de langue - lieu où s'exercent la compétence et la performance linguistique du traducteur - , alors que sur le plan du discours, où opère la traduction, il faudra relever les erreurs et lacunes du traducteur dans son exégèse du sens du TD. Ce sont évidemment les avatars de l'opération traduisante et non pas la méconnaissance des langues qui retiennent ici notre attention. $M$ ais la réalité linguistique est un continuum qui ne se laisse pas réduire à deux catégories hermétiquement cloisonnées, «faits de langue» et «faits de discours». Un premier exemple, qui relève de la connaissance de la langue ( «le génie de la langue»), illustrera la notion centrale, pour la discussion sur la prolixité des traductions, d'implicite et d'explicite.

Lorsqu'il y a choix binaire, le français n'exprime qu'un des deux pôles et laisse l'autre sous-entendu, alors que l'anglais formulera l'alternative dans sa totalité:

Do you want your bread plain ou toasted?

Ton pain, tu le veux grillé?

Ton pain, tu le veux grillé ou pas (grillé) ? est certes possible, mais, loin d'avoir le ton neutre et factuel de l'anglais, la question révélerait l'impatience du locuteur devant une réponse qui se fait attendre, ou supposerait que la question n'a pas été comprise du premier coup, etc.

«Tous les mots comptent», et ceux qui sont en trop ont des effets pervers sur I'intelligibilité des traductions. Dans l'exemple précédent, la version en apparence redondante créerait en fait une intention, un sens affectif qui n'existe pas dans leTD.

La redondance de la langue anglaise semble être une caractéristique qui se retrouve communément dans le discours; nous en avons vu plus haut un exemple dans un texte économique. Si le rédacteur anglais pouvait se dispenser de préciser ici ou là oil, la répétition «oil demand», «oil production», «oil shortage» qui parcourt le texte, loin de choquer le lecteur anglophone, semble s'imposer alors qu'elle lasserait très vite le lecteur francophone si elle devait être transposée dans le TA ${ }^{5}$, même si le traducteur utilisait l'artifice stylistique de la synonymie et des dérivés, pétrole/pétrolier, brut, ou noir. Dans un texte court (quelque 200 mots), dont l'unique propos est le pétrole, la réaction du lecteur francophone devant cette redondance oscillerait entre l'impatience et l'irritation, parce qu'il aurait des raisons de croire qu'en surprécisant, l'auteur (le traducteur) doute des facultés de compréhension immédiate de ses lecteurs.

Contrairement à ce qui se passe lorsque l'implicite de la langue est transgressé, lorsque l'implicite du discours n'est pas mis à profit, il n'y a pas de modification du sens du TD, mais «maladresse(s) » et autre(s) «lourdeur(s) » rédactionnelles ressenties à des degrés divers par le lecteur.

La répétition indue pourrait être attribuable à la confusion entre usage et fré quence textuelle. Or la nécessité d'employer le terme couramment utilisé dans le technolecte cible n'implique pas que ce terme doit se trouver autant de fois dans leTA que dans le TD ; c'est, chez les apprentis traducteurs surtout, " l'obsession des équivalents» (Bédard 1986 cité par Macklovitch 1996: 309), c'est-à-dire «la tendance à reproduire mécaniquement dans le texte cible les équivalents de tous les termes trouvés dans le texte source». La répétition indue dénote une compétence rédactionnelle insuffisante, par manque d'attention à la cohérence interne du TA (voir note 4).

À noter que l'implicite du discours peut à tout moment redevenir explicite; il revient au traducteur-rédacteur de doser la récurrence, soit pour éviter une ambi- 
guïté à un point donné du texte, soit pour rafraîchir la mémoire du lecteur dont quelque digression aurait pu distraire l'attention.

Les exemples d'inflation verbale qui suivent relèvent principalement d'une exégèse insuffisante du TD ou, plus généralement, d'une conception du sens qui réduit le TA, et par conséquent le TD, à la simple juxtaposition d'«unités de traduction » autonomes ou indépendantes (propositions ou phrases entières, voire paragraphes).

On sait que la compréhension s'appuie sur un acquis de connaissances générales ou, pour les domaines spécialisés, particulières. Or, le traducteur prolixe ne tient pas suffisamment compte de la situation qui a donné naissance à l'acte de communication. II semble oublier tout aussi fréquemment par ses redondances le savoir que le texte construit au fur et à mesure de son déroulement. Par ailleurs, tant que n'aura pas été saisi le caractère discontinu du sens, le traducteur cherchera une reformulation du TD dans des équivalences terme à terme, et il passera à côté des différentes façons aptes à désigner une même réalité. Par exemple, aucun mot ou tournure de l'anglais ne suggère immédiatement le français conjoncture, si courant en économie politique. D'où la difficulté, voire la réticence chez d'aucuns, à employer ce terme qui signifiera toute une proposition du TD. Dans l'exemple suivant, on imaginera sans peine les circonlocutions maladroites, la verbosité des traductions qui n'ont pas su se détacher de la formulation du texte source... et la perplexité provoquée par le corrigé du professeur :

If the hatmaker's prospects have generally changed because of circumstances special to hats [...]

Si sa clientèle diminue à cause de la conjoncture sectorielle [... ]

La connaissance du domaine et de la terminologie usuelle dans LA (conjoncture, sectoriel), la prise en compte de l'information véhiculée par le texte au point où apparait cette phrase (diminue plutôt que «change»), la vérification de la cohésion, de la cohérence interne du TA - c'est-à-dire la compétence rédactionnelle en LA (sa clientèle que permet la phrase précédente, plutôt que «la clientèle du chapelier ») et, finalement, l'exégèse qui conduit à comprendre, et donc à formuler, ce que signifie circumstances special to hats (la conjoncture sectorielle), sont autant de conditions nécessaires sans lesquelles les traductions ne peuvent être ni claires ni idiomatiques.

Deux exemples illustreront plus encore la prééminence de la situation pour arriver à une traduction précise et idiomatique. Pour le premier, il s'agit de la traduction d'un script qui accompagne le défilé de diapositives dans un cours audio-visuel:

Ce que vous avez fait doit ressembler à cette image proposait la traduction non révisée. L'anglais se devine aisément:

What you have done should look like this picture.

À l'impropriété près (qui passe souvent inaperçue en langue orale), la traduction pourrait passer pour acceptable. Une révision qui ne se préoccuperait que de cette incorrection donnerait:

Ce que vous avez fait doit correspondre à ce que vous voyez sur cette image

formulation lourde et maladroite qui, bien qu'irréprochable sur le plan de la langue, est aussi peu satisfaisante que la première version parce qu'elle ne tient pas pas compte de la dynamique du cours audio-visuel, de la situation qui impose la solution à laquelle aucune «traduction linguistique» ne pourrait à elle seule aboutir : 
Cette diapositive vous montre le connecteur [ou la pièce] en place.

Le second exemple (pancarte vue à l'aéroport de Londres) est emprunté à Pergnier (1980) :

Les passagers ne doivent pas savancer au-delà de ce point de contrôle sans avoir vérifié au préalable près de la compagnie avec laquelle ils voyagent.

Transcodage peu intelligible, a fortiori pour le voyageur anxieux ou en retard.

La formulation authentiquement française de la révision dit avec une grande concision toute l'information maladroitement explicite de la première traduction, mais combien plus clairement! Pour ce faire, la version révisée fait appel à deux formules administratives consacrées, accès réservé à et formalités de:

Accès réservé aux passagers ayant effectué les formalités d'embarquement.

II est intéressant de remarquer dans les deux versions les grandes différences entre les implicites et les explicites du français et de l'anglais, sans en tirer de conclusions générales et abusives sur «l'esprit des langues» et «la vision du monde» de leurs locuteurs, comme le fait la stylistique comparée. Cet exemple se distingue du précédent en ce qu'il relève davantage de la langue que du discours puisque la solution se trouve dans des expressions toutes faites (il serait plus exact de dire que c'est la situation qui permet de prévoir que le TA sera fait d'expressions consacrées: il s'agit d'un avis, comme on en trouve dans tous les aéroports du monde, destiné aux passagers; le support (la pancarte) oblige à une certaine concision, propre à chaque langue, et impose le recours à une série de formules consacrées).

\section{NATURE DE LA PROLIXITÉ ET CORRECTIFS}

Reprenons l'exemple relevé sur une boîte de lait en poudre pour mieux cerner la nature de la prolixité, c'est-à-dire ce qui constitue l'essentiel des différences entre la première traduction...

Ce produit est vendu au poids et non au volume. À cause de diverses manipulations le lait écrémé en poudre instantané peut se tasser dans l'emballage. C'est pourquoi le nombre de pintes obtenues en reconstituant le lait peut varier légèrement si l'on se sert d'une tasse à mesurer.

... et la révision:

Ces granulés sont vendus au poids et peuvent se tasser au cours de la manutention. La quantité de lait reconstitué peut varier selon que les granulés sont dosés au poids ou au volume.

- Prise en compte du contexte $;$; élagage des redondances:

a) Sur le plan du discours: la révision a supprimé dans la première phrase le redondant et non au volume, à cause des phrases qui suivent et du contexte (l'emballage sur lequel le poids est précisé). Si cette phrase avait été la seule indication du message, il est clair que et non au volume aurait été maintenu.

b) Sur le plan de la langue: suppression dans la deuxième phrase, pour des raisons encore plus évidentes, car ici c'est l'implicite de la langue qui a été transgressé (voir plus haut), de dans l'emballage, quasi-lapalissade. 
La manutention remplace l'impropre manipulations et la détermination maladroite, voire sibylline, de diverses (des diverses eût été plus clair), surprécison pour rendre l'anglais shipping dans shipping and handling. Dans une autre situation, la vente par correspondance par exemple, [coûts de] transport et [de] manutention s'imposerait bien évidemment.

- Prise en compte de la situation; nouveaux traits pertinents dans le TA de la réalité exprimée dans le TD - le «génie de la langue» cible:

Aspect bien évidemment central de la traduction (ou de la révision des textes prolixes), et difficile à diagnostiquer de prime abord avec autant de rigueur et d'objectivité que pour les catégories précédentes - où les corrections s'appuient largement sur les grammaires, les dictionnaires généraux et spécialisés et autre documentation authentique. Ici, c'est la compréhension plus ou moins immédiate, la bizarrerie de l'énoncé qui met le réviseur sur la piste. Les longueurs de la formulation ne sont plus de simples redondances lassantes, elles interfèrent avec la compréhension immédiate du texte. Le dernier élément de l'énoncé prolixe, si l'on se sert d'une tasse à mesurer, semble indiquer que pour qu'il n'y ait pas de «variations» dans le nombre de pintes obtenues en reconstituant le lait, il ne faut pas utiliser de tasse à mesurer (canadianisme pour «verre mesureur» ou «verre gradué»). Ce n'est pas faux, mais ce n'est pas l'optique de l'avertissement du TD qui tient d'abord à rassurer le consommateur qu'il en a bien pour son argent, même si la boîte n'est pas pleine, et qui indique les deux façons de reconstituer le lait, sachant que le verre mesureur se trouve dans tous les foyers, alors que la balance de ménage, plus précise que le précédent, y est moins répandue.

La prise en compte de la situation fait évidemment appel à la connaissance du domaine, connaissance non seulement encyclopédique mais aussi stylistique, parce que la compréhension du sujet doit s'accompagner nécessairement pour le traducteurrédacteur de la maîtrise du technolecte idoine, ce qui exige une lecture attentive des textes authentiques pour en imiter le style.

Mesurer la proportion des ingrédients, c'est doser au poids ou au volume, quel que soit l'instrument utilisé pour ce faire. D'où la disparition, dans la version révisée, du référent tasse à mesurer, qui n'est pas le «propos» du TD, faut-il préciser pour quiconque y verrait une omission ou un à-peu-près de la traduction.

L'analyse qui précède trouve son utilité dans l'enseignement de la traduction à quelque niveau de l'apprentissage que ce soit, particulièrement lorsqu'elle permet de cerner les erreurs, lourdeurs et maladresses qui ne trouvent pas d'explication dans les dictionnaires, les grammaires ou autres ouvrages de référence. $M$ ais cette approche descriptive et curative appelle son pendant de mesures préventives pour que la classe de traduction soit pleinement profitable.

\section{COMMENT PRÉVENIR (OU CORRIGER) LA PROLIXITÉ $Q$ uelques procédés pédagogiques}

Les procédés qui suivent visent à aider les étudiants à produire des traductions exemptes de prolixité, et à ce titre méritent de leur être présentés dès le début de l'apprentissage. Le professeur devra bien sûr y revenir à tous les niveaux du cursus et insister sur tel aspect plutôt que tel autre en fonction de la gradation des cours et du 
degré de spécialisation des textes. Tout comme la typologie des textes conduit à la hiérarchisation des fonctions descriptive, argumentative, émotive, etc., les procédés qui suivent prendront plus ou moins d'importance et de temps dans le travail préparatoire à la formulation du TA selon le texte à traduire. Le lecteur de ces lignes comprendra aussi que ces procédés sont en quelque sorte l'envers des correctifs étudiés précédemment; ceux-ci et ceux-là sont interchangeables dans la mesure où tout travail de traduction devrait comporter une phase de vérification en tous points semblable à une révision professionnelle. Le professeur les utilisera tant dans ses corrections que dans les exercices de traduction faits en classe.

La traduction en classe se déroulera de la façon suivante:

\section{- Le résumé: à la recherche du thème et du propos ${ }^{7}$}

Pour s'assurer que le texte est compris, rien de tel que le résumé, à faire oralement dans la langue d'arrivée. Je ne m'étendrai pas sur ce procédé classique et éprouvé, bien que trop souvent négligé ou insuffisamment exploité. Savoir hiérarchiser les éléments du texte développe la capacité analytique et critique de l'étudiant. Pour que le résumé ne prenne pas la forme d'une longue paraphrase, demander successivement de trouver un titre aux paragraphes du TD, puis, pour les regrouper, aux différents mouvements du texte, et enfin réduire le résuméà une manière de titre assorti d'un sous-titre (le titre du TD n'est jamais fourni au début de l'exercice, non parce qu'il fournirait la solution, bien au contraire. Les titres posent des difficultés particulières de traduction car, loin d'être des condensés informatifs, ce sont plus souvent des énoncés énigmatiques, amusants ou accrocheurs, où abondent fréquemment les jeux de mots). Cet exercice est particulièrement important dans I'optique du présent article; car réduire un texte à l'essentiel, surtout s'il est argumentatif, permet de mieux faire distinguer le ou les thèmes du propos, sans quoi, nous venons de le voir, la prolixité a le champ libre. En outre, l'argument principal bien cerné réduira les risques de contresens et les faux sens graves où font souvent tomber les tournures grammaticales ou idiomatiques du TD inconnues de l'étudiant.

À cette première étape, la rigueur terminologique n'est pas exigée des étudiants.

\section{- Le point sur les connaissances dans le domaine: l'objet du texte}

a) sur le plan non linguistique: réunir les connaissances, souvent éparses et dé cousues, même lorsque les étudiants sont censés s'être préparés, et les structurer. Cette phase recoupe souvent la précédente ${ }^{8}$, car le résumé fait apparaître de nombreuses incompréhensions, par méconnaissance du domaine ${ }^{9}$, qu'il convient de corriger sans attendre. En profiter pour situer le texte et son argumentation s'il y a lieu : texte narratif, explicatif, ou qui expose une problématique qui remet plus ou moins en question les connaissances généralement admises (d'où l'importance, dans ce dernier cas, de bien cerner ce qui est communément connu du domaine par les destinataires du TD et du TA, la similitude entre les deux ne devant pas être présupposée). En résumé, pour quels destinataires le TD a-t-il été écrit, et pour leur apprendre quoi de plus que ce qu'ils doivent déjà savoir pour être en mesure de comprendre le TD ? ( Question à reposer pour les destinataires du TA.)

b) sur le plan terminologique: le jeu de questions doit amener les étudiants à fournir les termes nécessaires à la traduction ; phase assez rapide car la recherche aura été faite avant le cours, mais qui oblige plus d'une fois à revenir sur la phase 
précédente, les termes erronés qui sont proposés révélant une méconnaissance du domaine.

\section{- L'analyse détaillée du sens: la part de l'implicite et de l'explicite}

C'est cette troisième étape qui doit conduire à éviter les formulations prolixes (ou à les éliminer s'il s'agit d'un travail de révision) ; elle ne saurait évidemment pas se passer des précédentes. Elle s'applique tant au texte de départ qu'à la relecture de la traduction, et elle porte sur deux niveaux, ceux de la langue et du discours ${ }^{10}$.

Le travail consistera à distinguer le savoir sur lequel repose l'énoncé - préalable au texte, mais aussi celui que le texte construit au fur et à mesure de son déroulement - de l'information nouvelle contenue dans chaque mouvement du texte (phrase ou groupe de phrases constituant un [sous-]propos). Cette «explication de texte» se faisant dans la langue d'arrivée, elle permettra de faire ressortir ce qui serait pour le TA redondant (discours), pléonastique (langue), ou, à l'inverse, les non-dits et implicites du TD qu'il faudra énoncer.

\section{- Relecture/révision : I'exploitation des documents authentiques}

Dernière étape, et étape préliminaire aussi. L'exploitation des documents authentiques devrait en effet précéder toute traduction, générale ou spécialisée ${ }^{11}$, car la lecture de documents judicieusement choisis dans la langue cible servira à se familiariser à la fois avec le domaine et avec le technolecte.

Le sujet mériterait de plus longs développements que ne le permet le cadre de cet article. À défaut, ces quelques remarques générales: I'exploitation stylistique du texte authentique, au-delà de la recherche terminologique à laquelle les étudiants se cantonnent, est ardue, il faut le reconnaître, et demande l'accompagnement du professeur. Le professeur aura le choix de laisser les étudiants libres dans leur recherche de documents ou de les guider s'ils ne savent pas encore séparer le bon grain de l'ivraie. Quelques séances de travail seront nécessaires pour montrer que le texte authentique fournit bien plus que des termes, que l'environnement de ces termes contribue tout autant que ceux-ci au caractère sui generis du texte, et que le texte authentique fournit bien mieux que le dictionnaire bilingue le verbe, le complément, le qualificatif idoines sans lesquels le style de la traduction sera, au mieux, grammaticalement correct mais paraphrastique, enfantin, vide de tournures idiomatiques, et au pire, un calque stylistique du TD émaillé d'impropriétés et d'images boiteuses dont l'intelligibilité pâtira.

Pour illustrer les trois premières étapes, reprenons brièvement notre exemple du lait en poudre, non plus comme travail de révision, mais dans l'optique de la traduction:

This product is sold by weight and not by volume. The powder can settle or pack down during the shipping and handling. If a measuring cup is used to do the mixing, you may not always obtain exactly the same number of quarts of milk from the contents of this package.

Le résumé (étape 1) pourrait conduire à une formulation proche de la forme définitive! La deuxième étape (le point sur les connaissances dans le domaine, I'objet du texte), notamment la question qui la résume (pour quels destinataires le TD a-t-il été écrit, et pour leur apprendre quoi, etc. ?) conduit 1) à la réponse: le produit est vendu au poids, seule mesure qui donnera des résultats constants (ou : mesuré au volume, le produit donnera des résultats variables) ; 2) à la recherche des termes exacts: lait en 
poudre, certes, mais si ce thème devient sous-propos ( «product » devenant «the powder can settle... »), «la poudre» ne peut se dire sans être qualifiée. Or, «la poudre de lait [ou : le lait en poudre] peut se tasser » est une formulation qui donnerait l'impression d'énoncer les caractéristiques scientifiques ou inhérentes de ce produit. D'où le nécessaire «granulés». M anutention et transport seront énoncés, mais il sera sans doute plus facile de faire comprendre à l'étape suivante que transport n'apporte aucune information utile dans ce contexte.

La troisième étape permettra de déceler le doublet settle or pack down, et donc ce qui ne pourrait être qu'un pléonasme en français. Sur le plan du discours, les étapes précédentes, surtout si elles sont bien encadrées, auront très probablement conduit les étudiants à éliminer progressivement de leurs réponses toute référence à l'instrument de mesure (le verre mesureur) ; sinon, c'est la quatrième étape, la relecture/ révision, qui devra le faire disparaitre (voir également ci-dessus ${ }^{12}$ ).

\section{CONCLUSION}

La longueur, lorsqu'elle est manifeste, du texte traduit par rapport à l'original ne se laisse pas mesurer aisément. Non seulement les différents critères possibles (nombre de mots, nombre de caractères-espaces, ou nombre de phonèmes) donnent des résultats contradictoires mais, le décompte fait, l'équivalence ou la non-équivalence quantitative des textes source et cible ne permet pas à elle seule de porter un jugement qualitatif sur tel passage ou sur le TA dans son ensemble. La longueur patente peut tout au plus être la première indication, pour le réviseur comme pour l'étudiant, d'une traduction probablement déficiente. Si cette déficience était un simple manque de concision, le reproche serait mineur et les corrections simples (il suffirait de biffer les mots en trop et de raccorder les éléments retenus). Mais nous avons vu que ces traductions souffraient plus par leurs longueurs (prolixité) que par leur étendue, et que cette verbosité nuisible à l'intelligibilité révélait un certain nombre de manques à plusieurs niveaux: la connaissance du domaine et de son technolecte en LA (par sa stylistique autant que par sa terminologie), l'exégèse du TD, la prise en compte de la situation qui a donné naissance au message, la cohésion et la cohérence du TA. Les corrections qui s'imposent conduisent le plus souvent à une reformulation d'envergure, parfois complète, chaque fois que les ressources et les caractéristiques du technolecte d'arrivée ont été sous-exploitées.

Une conclusion s'impose: la prolixité en traduction ne ressemble nullement à ce qu'elle est en langue spontanée - délayage, explications «en long et en large», inflation de «points sur les $\mathrm{i}$ ». Loin de rendre le message limpide, la verbosité traductionnelle brouille le sens ou en ralentit l'appréhension.

On a dit que trop de notes nuisent à la bonne musique; cela pourrait s'appliquer mutatis mutandis à la traduction, mais le hic c'est que cette maxime ne révèle pas comment faire la part des choses et trouver la note juste, le phrasé (la phraséologie) et le ton de la composition. S'agissant de «bonnes traductions» (c'est-à-dire intelligibles et idiomatiques), cet article a voulu montrer que c'est en remontant aux fondements de l'opération traduisante que l'on peut espérer trouver quelques clés. 


\section{NOTES}

1. Dans les six combinaisons de trois langues: Barth (1961). Également de l'italien au français: Monjo (1973).

2. Durieux (1990: 55-60): «... la Société française des traducteurs recommande aux traducteurs [de] tenir compte dans leurs devis [...] pour la traduction d'anglais en français [d']un coefficient de foisonnement courant de 25 pour cent. »

3. Cette reclassification des «cinq causes les plus fréquentes» énoncées par Durieux a pour but de mieux distinguer les fautes qui relèvent de l'analyse du sens et celles qu'une plus grande pratique de la rédaction devrait permettre d'éliminer. Je n'ai pas retenu comme causes distinctes le transcodage et la totalité du sémantisme, car sur la base des exemples qu'en donne Durieux, ces deux causes peuvent rentrer respectivement dans ses catégories calque ou pléonasme/redondance d'une part, et surtraduction d'autre part.

4. La recherche de la concision optimale relève de la compétence de l'étudiant en rédaction française et non pas de son apprentissage de la traduction en tant que telle. Pour important qu'il soit dans tous les cours de traduction générale ou spécialisée, l'enseignement systématique de la rédaction ne peut s'y insérer et doit relever par conséquent d'un ou plusieurs cours parallèles de rédaction française.

5. Les meilleures copies d'étudiants ont réduit l'occurrence à cinq, sur les huit du TD, là où le corrigé s'est contenté de deux.

6. Bien faire distinguer aux étudiants la différence entre contexte (le texte-message pris globalement) et situation (I'extra-linguistique qui a donné naissance au message et dans lequel celui-ci s'inscrit).

7. Terminologie de la Stylistique comparée.

8. Dans le cas de textes spécialisés, on aurait avantage à commencer par cette mise au point avant le résumé.

9. Domaine au sens large ici, car il peut tout aussi bien s'agir d'une méconnaissance de l'actualité, d'un fait de culture, etc.

10. Selon leniveau du cours, le professeur aura peut-être avantage à employer, s'agissant du discours, dit et non-dit, pour réserver implicite et explicite à la langue.

11. Termes relatifs on le sait, d'autant qu'il n'est guère de textes dits «généraux» qui ne fassent appel à un point donné, lorsqu'il s'agit de les traduire surtout, à des connaissances «pointues». Sur le plan pédagogique, les textes «généraux» que nous donnons en première année présentent à toutes fins pratiques le même degré de difficulté stylistique que les textes «spécialisés» des $2^{\mathrm{e}}$ et $3^{\mathrm{e}}$ années; le modèle authentique est donc tout aussi indispensable et il s'exploite avec le même profit.

12. II convient de préciser que ces remarques doivent s'appliquer avec souplesse pour tenir compte d'autres traductions possibles, sachant qu'il y a souvent plus d'une façon de bien dire les choses et rarement une unique solution (celle du professeur?) à laquelle les étudiants devraient absolument aboutir pour être déclarés bons traducteurs.

\section{RÉFÉRENCES}

Barth, G. (1961) : Recherches sur la fréquence et la valeur des parties du discours en français, en anglais et en espagnol, Paris, Didier.

Bédar d, Claude (1986) : La traduction technique: principes et pratique, Montréal, Linguatech.

Dur ieux, Christine (1990) : "Le foisonnement en traduction technique d'anglais en français», M eta, 35 (1), p. 55-60.

M ack I ovitch, Elliott (1996) : «Peut-on vérifier automatiquement la cohérence terminologique?», M eta, 41 (3), p. 299-316.

M onjo, A. (1973) : «Exégèse et re-création dans la traduction poétique», Études de linguistique appliquée, $n^{\circ} 12$, Paris, Didier.

Pergnier, Maurice (1980): Les fondements sociolinguistiques de la traduction, Paris, Honoré Champion. 